\section{HIGH PREVALENCE OF UNDIAGNOSED CARDIAC DYSFUNCTION IN THE OLDEST OLD: FINDINGS FROM THE NEWCASTLE 85+ STUDY}

doi:10.1136/heartjnl-2011-300198.98

${ }^{1} \mathrm{~F}$ Yousaf, ${ }^{1} \mathrm{~J}$ Collerton, ${ }^{2} \mathrm{~A}$ Kenny, ${ }^{1} \mathrm{~T}$ Kirkwood, ${ }^{1} \mathrm{C}$ Jagger, ${ }^{1} \mathrm{~A}$ Kingston, ${ }^{3} \mathrm{~B}$ Keavney. ${ }^{1}$ Institute of Ageing and Health, Newcastle University, Newcastle upon Tyne, UK; ${ }^{2}$ Freeman Hospital, Newcastle upon Tyne, UK; ${ }^{3}$ Institute of Human Genetics, Newcastle University, Newcastle upon Tyne, UK

Background Heart failure prevalence increases sharply at older ages. The section of the population aged 85 and over represents the most rapidly increasing demographic worldwide. Previous epidemiological studies of ventricular dysfunction and heart failure have included only small numbers of the "oldest old", and have generally been conducted in hospital-based settings, potentially introducing ascertainment biases. We conducted a community-based study of the oldest old using domiciliary echocardiography to estimate the prevalence of cardiac dysfunction and heart failure. Since in elderly people with multiple comorbidities, heart failure may more frequently be incorrectly diagnosed, we cross-referenced our findings to preceding diagnoses present in general practice records.

Methods Four hundred and twenty-seven individuals aged 86-89 years (mean age 87.9 years; 39.1\% $(n=167)$ men, $60.9 \%$ $(n=260)$ women) were visited in their usual place of residence. A full cardiovascular and medical history, including current medication, was taken; symptoms were graded using the NYHA classification. Previous diagnoses of heart failure (HF) were abstracted from the GP record. Participants underwent 2-D and Doppler echocardiography, including tissue Doppler measurements of LV long axis velocities, using a portable instrument (Vivid-I, GE Healthcare). LV systolic and diastolic dysfunction were graded according to American Society of Echocardiography guidelines.

Results LV systolic function could be quantified in 93.2\% ( $n=398)$ participants and diastolic function (classified as normal, mild, moderate or severe dysfunction) in $88.1 \%(n=376) .37 .2 \%$ of participants $(n=140 / 376)$ had normal cardiac function or isolated mild diastolic dysfunction; $19.6 \%(n=78 / 398)$ had moderate or severe LV systolic dysfunction, which was commoner in men (27.4\%) than women $(14.5 \%)$; and $14.4 \%(n=54 / 376)$ had isolated moderate or severe diastolic dysfunction. $65.1 \%$ (278/427) of participants had valid data on previous diagnosis of HF, NYHA class and echocardiographic assessment of cardiac dysfunction. Of these, 37.4\% (104/278) had clinical evidence of HF, which was defined as NYHA class II, III, or IV symptoms with underlying systolic dysfunction $(29.5 \%(82 / 278))$ or isolated moderate or severe diastolic dysfunction $(7.9 \%(22 / 278))$ on echo. Only $7.6 \%(21 / 278)$ had a previous diagnosis of HF. $33.1 \%(n=92 / 278)$ had no previous diagnosis of HF but had clinical evidence of HF and an additional $21.6 \%$ ( $n=60 / 278$ ) had no previous diagnosis but evidence of pre-clinical HF (NYHA class I with systolic or moderate/severe diastolic dysfunction). Of those with a previous diagnosis of HF, $23.5 \%$ $(n=5 / 21)$ had no echocardiographic evidence of cardiac dysfunction.

Conclusions Systolic and diastolic dysfunction and HF were commoner in our population than previous studies in the "younger old" have suggested. There are significant levels of both undiagnosed and misdiagnosed HF in this age group.

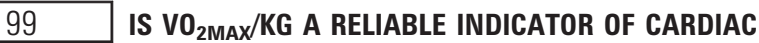 DYSFUNCTION IN OVERWEIGHT HEART FAILURE PATIENTS?}

doi:10.1136/heartjnl-2011-300198.99

S Chinnappa, N Lewis, D Barker, L B Tan. Leeds Teaching Hospitals NHS Trust, Leeds, UK

Background Peak $\mathrm{O}_{2}$ consumption $\left(\mathrm{Vo}_{2 \max }\right)$ of $\leq 14 \mathrm{ml} / \mathrm{kg} / \mathrm{min}$ has been widely accepted as being indicative of poor cardiac function warranting consideration for transplantation (Circulation 2010; 122:173). We examined whether this variable is a good indicator of cardiac function in overweight heart failure (HF) patients.

Methods We compared the cardiopulmonary exercise performance and non-invasive haemodynamics of overweight (BMI $>34 \mathrm{~kg} / \mathrm{m}^{2}$ ) and non-overweight (BMI $\leq 30)$ male heart failure patients in NYHA Classes II and III, with those of healthy male volunteers with no known cardiovascular diseases ( $n=101$, age $43.2 \pm 18.1$ (SD) years, BMI $26.0 \pm 3.1$ ) as controls. Their physical and cardiac functional reserves were measured during treadmill exercise testing with standard respiratory gas analyses and rebreathing method of non-invasively measuring cardiac outputs during peak exercise.

Results Consecutive overweight HF were screened and 24 patients (age

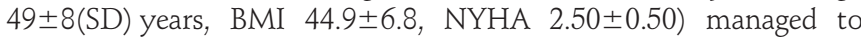
exercise to acceptable cardiopulmonary limits (peak $R E R=1.01 \pm 0.12$ ), and achieved $\mathrm{Vo}_{2 \max }$ of $16.8 \pm 4.6 \mathrm{mls} / \mathrm{kg} / \mathrm{min}$ which was significantly lower than controls $\left(37.0 \pm 10.7 \mathrm{mls} / \mathrm{kg} / \mathrm{min}, \mathrm{p}<10^{-6}\right)$ and also lower than those of 30 non-overweight HF counterparts $(20.0 \pm 3.7 \mathrm{mls} / \mathrm{kg} /$ min, $\mathrm{p}=0.0019$, age $49 \pm 15$ years, BMI 25.0 \pm 2.9 , NYHA 2.48 \pm 0.51 ). As shown in Abstract 99 figure 1, the overweight HF patients had $\mathrm{VO}_{2 \max }$ values which distributed around the $14 \mathrm{mls} / \mathrm{kg} / \mathrm{min}$ cut-off value, and 9 of whom were indeed below this cut-off value. However, the uncorrected $\mathrm{Vo}_{2 \max }$ were higher than those of non-overweight counterparts (Overweight: $2575 \pm 748$ vs $1594 \pm 325 \mathrm{mls} / \mathrm{min}, \mathrm{p}<10^{-6}$ ), and its range of $1485-4210 \mathrm{mls} / \mathrm{min}$ overlapped with the range of $1244-5774 \mathrm{mls} / \mathrm{min}$ in controls. The peak cardiac power output $\left(\mathrm{CPO}_{\max }, 4.5 \pm 1.6 \mathrm{~W}\right.$, minimum $\left.2.7 \mathrm{~W}\right)$ of overweight $\mathrm{HF}$ patients were clearly above those of non-overweight $\left(2.4 \pm 0.6 \mathrm{~W}, \mathrm{p}<10^{-6}\right.$, Abstract 99 figure 2) and all above the transplant cut-off value of $1.5 \mathrm{~W}$.

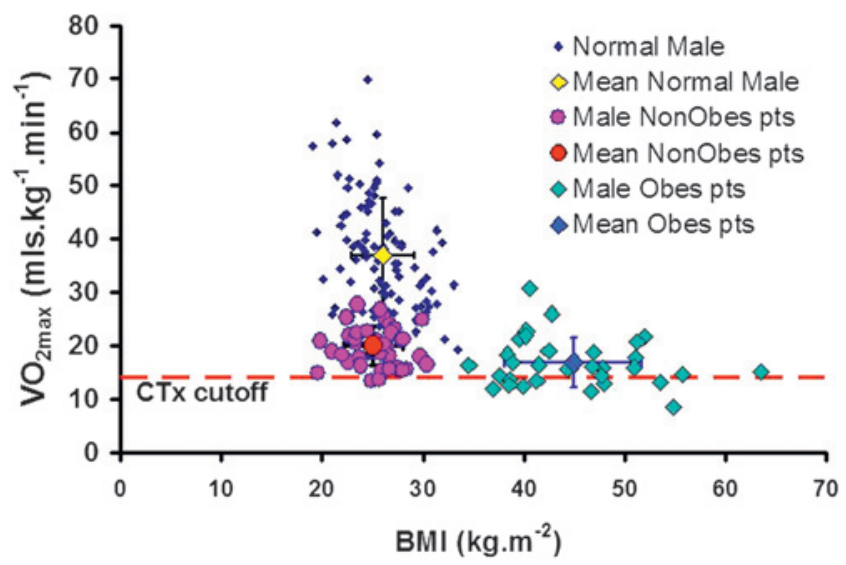

Abstract 99 Figure 1

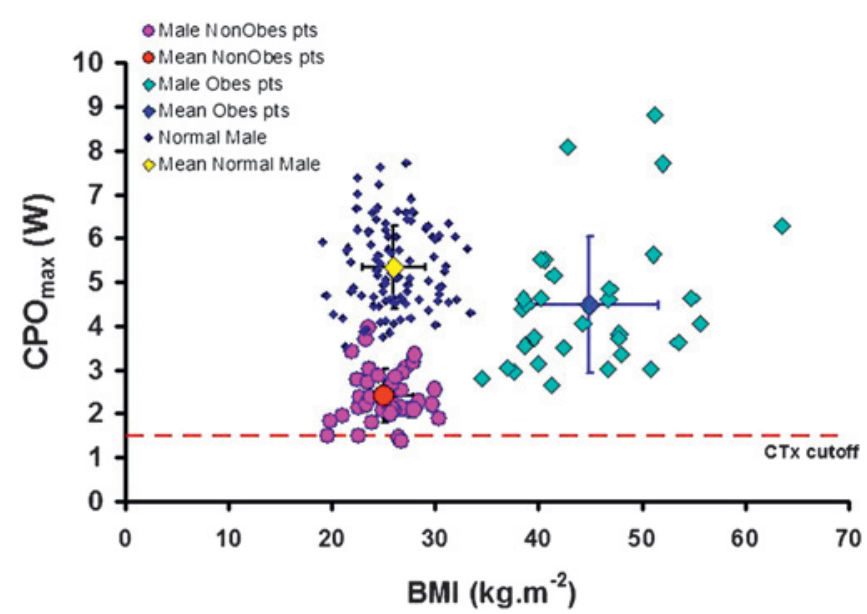

Abstract 99 Figure 2 
Conclusion These results indicate that in principle $\mathrm{VO}_{2 \max }$ in $\mathrm{ml} / \mathrm{kg} / \mathrm{min}$ as an indirect indicator of cardiac function or for cardiac transplantation selection is unreliable when applied to overweight heart failure patients. Extending this concept to the entire spectrum of body weights, the practice of correcting $\mathrm{Vo}_{2 \max }$ by body weight in cardiological practice would also require urgent reconsideration.

\section{PRESSURE VS FLOW AS A GUIDE FOR PACEMAKER OPTIMISATION? THE ACUTE HAEMIODYNAMIC EFFECTS OF CHANGES TO ATRIOVENTRICULAR DELAY}

doi:10.1136/heartjn|-2011-300198.100

C H Manisty, B Unsworth, R Baruah, P Pabari, Z I Whinnett, J Mayet, D P Francis. Imperial College, St. Marys Hospital, London, UK

Background Non-invasive blood pressure monitoring by continuous finger photoplethysmography (Finometer) may have value in pacemaker

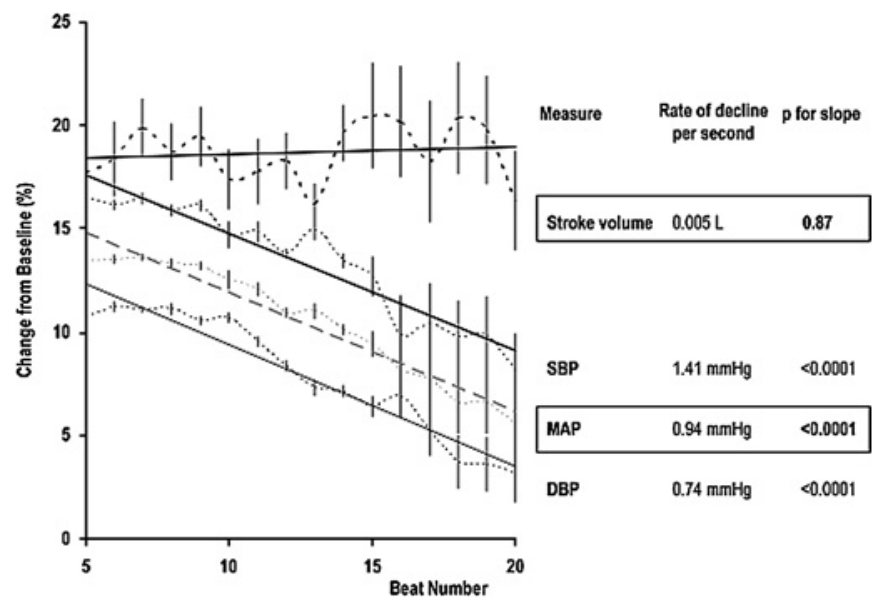

Abstract 100 Figure 1

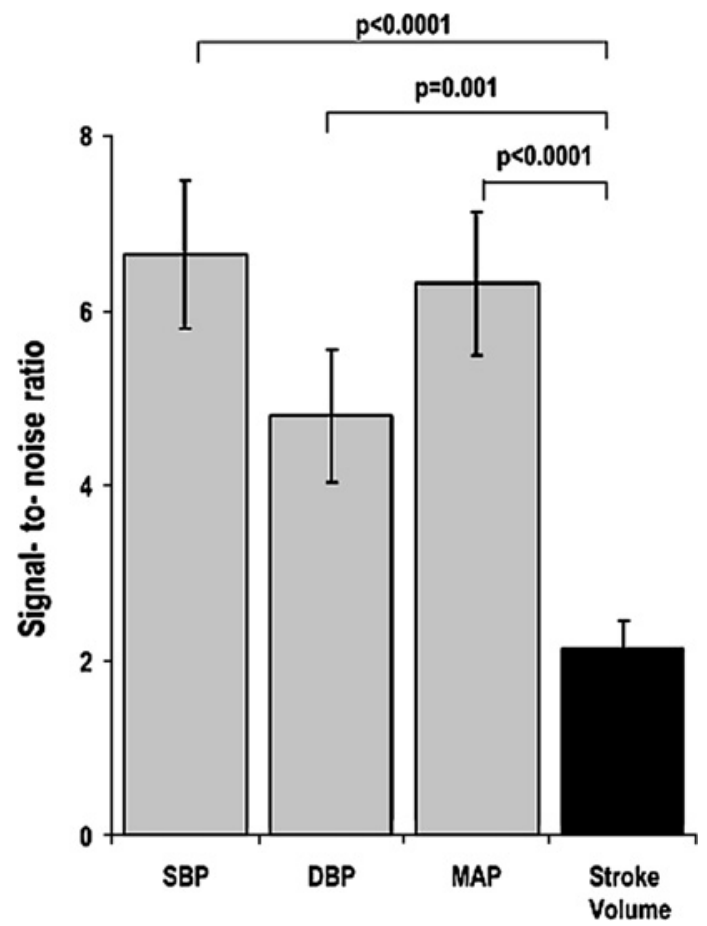

Abstract 100 Figure 2 optimisation. However, the immediate increment in blood pressure seems to diminish somewhat in the initial minute: it is unclear whether this is due to an (undesirable) fall in stroke volume or a (desirable) compensatory reduction in peripheral resistance.

Methods and Results We studied this question by measuring beat-bybeat stroke volume (flow) using Doppler echocardiography, and blood pressure using continuous finger photoplethysmography, during and after atrioventricular delay adjustment from 40 to $120 \mathrm{~ms}$ in 19 subjects with cardiac pacemakers. Quintuplicate experimental runs were performed. Blood pressure and stroke volume (flow) both increased immediately $(\mathrm{p}<0.00001$ within one heartbeat). The immediate pressure increment correlated strongly with the immediate flow increment $(r=0.74, p=0.0001)$. Pressure showed a partial decline a few seconds later (average rate $0.65 \mathrm{~mm}$ $\mathrm{Hg} /$ beat, $\mathrm{r}=-0.98, \mathrm{p}<0.0001$ ), in contrast, flow did not decline $(\mathrm{p}=\mathrm{NS})$, Abstract 100 figure 1. Signal-to-noise ratio was significantly better for pressure than flow $(6.3 \pm 3.6$ vs $2.1 \pm 1.4, \mathrm{p}<0.0001)$, Abstract 100 figure 2

Conclusions Improving atrioventricular delay immediately increases blood pressure; however this effect decays slightly over the subsequent minute. This is due to compensatory vasodilatation rather than a reduction in cardiac function. Pressure changes are simpler to measure and easier to distinguish from random variation than Doppler measurements of flow, but are best measured immediately, before the vascular compensation.

\section{WHAT DEGREE OF PULMONARY HYPERTENSION PREDICTS POOR OUTCOME IN PATIENTS WITH LEFT VENTRICULAR SYSTOLIC DYSFUNCTION? A 10-YEAR FOLLOW-UP STUDY}

doi:10.1136/heartjnl-2011-300198.101

${ }^{1} \mathrm{~B}$ R Szwejkowski, ${ }^{1} \mathrm{D}$ H J Elder, ${ }^{1} \mathrm{~A}$ M J Choy, ${ }^{2}$ S D Pringle, ${ }^{1} \mathrm{~A}$ D Struthers, ${ }^{1} \mathrm{C}$ C Lang. ${ }^{1}$ University of Dundee, Dundee, UK; ${ }^{2}$ Department of Cardiology, NHS Tayside, Dundee

Introduction The presence of pulmonary hypertension in left ventricular systolic dysfunction (LVSD) is an ominous sign. It remains unclear the level at which pulmonary hypertension conveys a mortality risk in patients with LVSD.

Methods We performed a record-linkage study in Tayside, UK (population approximately 400000 ) utilising the Tayside echocardiogram database ( $>100000$ echo's) maintained by the Health Informatics Centre (HIC). Datasets from HIC include mortality data and other health care activities linked anonymously by the community health index (CHI) number. Patients were included in the analysis if they had LVSD and had a right ventricular systolic pressure (RVSP) measurement. Cox proportional hazards regression analysis was used to examine the effects of different ranges of RVSP measures on all cause mortality.

Results 2910 patients (mean age, $74.5 \pm 11.4$ years; $43 \%$ male) met entry criteria. Mean RVSP was $43.3 \pm 12.7 \mathrm{~mm} \mathrm{Hg}$ and median follow was 362 days (IOR 129-850 days). There was a significant correlation between RVSP and survival $(p<0.0001)$. In quartiles of RVSP, the HR after adjustment for confounding factors including LVSD and the presence of chronic obstructive pulmonary disease (COPD) were: RVSP 35-41 mm Hg, HR 1.12 (95\% CI 0.95 to 1.32, $\mathrm{p}=0.175$ ), RVSP 42-50 mm Hg, 1.27 (1.07 to 1.49, $\mathrm{p}<0.001$ ) and RVSP 51-106 mm Hg 1.62 (1.38 to 1.1, p<0.001). For each $5 \mathrm{~mm}$ $\mathrm{Hg}$ stepwise increase in RVSP the HR for all cause mortality was 1.07 (1.04 to $1.09, \mathrm{p}<0.001$ ). Abstract 101 figure 1 shows the Kaplan-Meier survival curves for all cause mortality for all patients expressed as different RVSP quartiles. 